\section{Genome Resources of Four Distinct Pathogenic Races Within Fusarium oxysporum f. sp. vasinfectum that Cause Vascular Wilt Disease of Cotton}

\author{
Subodh K. Srivastava, ${ }^{1,2}$ Kurt A. Zeller, ${ }^{1, \dagger}$ James H. Sobieraj, ${ }^{1,2}$ and Mark K. Nakhla ${ }^{1}$ \\ ${ }^{1}$ USDA-APHIS-PPQ, S\&T-Beltsville Laboratory, Beltsville, MD 20705 \\ ${ }^{2}$ Department of Entomology and Plant Pathology, North Carolina State University, Raleigh, NC 27695
}

\begin{abstract}
Whole genome sequence (WGS) based identifications are being increasingly used by regulatory and public health agencies to facilitate the detection, investigation, and control of pathogens and pests. Fusarium oxysporum f. sp. vasinfectum is a significant vascular wilt pathogen of cultivated cotton and consists of several pathogenic races that are not each other's closest phylogenetic relatives. We have developed WGS assemblies for isolates of $F$. oxysporum f. sp. vasinfectum race 1 (FOV1), race 4 (FOV4), race 5 (FOV5), and race 8 (FOV8) using a combination of Nanopore (MinlON) and Illumina sequencing technology (Mi-Seq). This resulted in assembled contigs with more than $100 \times$ coverage for each of the $F$. oxysporumf. sp. vasinfectum races and estimated genome sizes of FOV1 $52 \mathrm{Mb}$, FOV4 $68 \mathrm{Mb}$, FOV5 $68 \mathrm{Mb}$, and FOV8 $55 \mathrm{Mb}$. The AUGUSTUS gene prediction program predicted 16,263 genes in FOV1, 20,259 genes in FOV4, 20,375 genes in FOV5 and 16,615 genes in FOV8. We were able to identify 525 genes unique to FOV1, 570 unique to FOV4, 1,242 unique to FOV5, and 383 unique to FOV8. We expect that these findings will help in comparative genomics and in the identification of unique genes as candidate targets for diagnostic marker and methods development to permit rapid differentiation of $F$. oxysporum $\mathrm{f}$. sp. vasinfectum subgroups.
\end{abstract}

\section{Genome Announcement}

Fusarium oxysporum f. sp. vasinfectum is a fungal pathogen of cultivated cotton (Gossypium sp.) that causes a damaging vascular wilt disease. This pathogen was first identified in 1892 causing a wilt disease of cotton in Alabama (Davis et al. 2006), but has a global distribution. F. oxysporum f. sp. vasinfectum infection can result in significant economic losses to cotton growers, especially when a field is also infested with the cotton root-knot nematode, Meloidogyne incognita (Davis et al. 2006). F. oxysporum f. sp. vasinfectum can persist in infested soils due to its chlamydospore stage, or in a disease-free association with the root systems of other plant species. F. oxysporum f. sp. vasinfectum can also infect cotton seed and may be transported to new areas by local and international seed trade. F. oxysporum f. sp. vasinfectum can be subdivided into pathogenic races if specific sets of cotton cultivars are used as differentials (Armstrong and Armstrong 1960). Up to eight pathogenic races have been described, although other data suggests that a few of those races are not actually distinct from each other. Phylogenetic analyses and DNA sequence data from housekeeping genes has been used to subdivide members of these pathogenic races into four distinct phylogenetic subgroups (Skovgaard et al. 2001). These four phylogenetic subgroups are not necessarily each other's closest relatives or even derived from a single ancestral source geographically, or

${ }^{\dagger}$ Corresponding author: K. A. Zeller; kurt.a.zeller@usda.gov

The author(s) declare no conflict of interest.

Accepted for publication 22 August 2020.

\section{Funding}

This research was supported by USDA AQI funds provided the S\&T Beltsville laboratory. This research was supported in part by the intramural research programs of the U.S. Department of Agriculture/Animal and Plant Health Inspection Service (APHIS)/Plant Protection and Quarantine (PPQ). The findings and conclusions in this preliminary publication have not been formally disseminated by U.S. Department of Agriculture and should not be construed to represent any agency determination of policy.

\section{Keywords}

computational biology, fungal pathogens, pathogen detectiondius 
phylogenetically. However, each of these pathogenic races causes a similar range of disease symptoms on cultivated cotton.

F. oxysporum f. sp. vasinfectum pathogenic race 4 (FOV4) is of greatest concern to U.S. agriculture as many of its isolates can cause significant disease in cotton in the absence of the cotton root-knot nematode (Kim et al. 2005). FOV4 is believed to have originated in Asia but has been known within in the United States from California since at least 2003, and was first identified in some cotton-growing areas of Texas during 2017 (Halpern et al. 2018). Monitoring the spread of FOV4 and limiting its impact on cotton growing regions within the United States are priority issues for the U.S. cotton industry.

In order to help develop molecular diagnostics methods to rapidly differentiate among these races and phylogenetic subgroups, we have generated draft genome sequences for single members of each of the four phylogenetic subgroupings as defined in Skovgaard et al. (2001), and are using these reference genomes to begin to characterize unique genes and genomic regions in each subgroup to serve as targets for novel diagnostics assays. We obtained the isolates used in this work from the ARS Culture Collection (NRRL) in Peoria, IL. We prepared genomic DNA for our reference isolates by placing small plugs of colonized media from potato dextrose agar plates into flasks of potato dextrose broth. We allowed cultures to grow at room temperature $\left(22\right.$ to $\left.24^{\circ} \mathrm{C}\right)$ for 3 days on an orbital shaker set to $100 \mathrm{rpm}$. After 3 days, we harvested mycelia by gravity filtration through KenAG Milk Filters, patted most of the moisture out of the retained filtered material with clean paper towels, and placed pieces of these filtered cultures into two to four Lysing Matrix A tubes (MP Biomedicals). We held these tubes at $-20^{\circ} \mathrm{C}$ until needed for DNA isolations. For DNA isolations, we disrupted the tissue using a FastPrep24 tissue homogenizer, and then extracted DNA from these materials with DNeasy Plant Mini Kits (Qiagen) using the manufacturers standard protocols. The Illumina (Mi-Seq) genomic DNA libraries using the Nextera DNA Flex Library Prep Kit protocol provided by Illumina was constructed as per manufacture standard protocol. The library quality was evaluated using the Agilent Genomic Screen Tape Assay (Agilent Technologies). These libraries were sequenced on Mi-Seq version 3.0 reagent kit produced 300-bp pair ends reads and generated around 20 million reads for each race as per standard protocol (Rivera et al. 2018).

We used 500 to $600 \mathrm{ng}$ of DNA from each isolate for preparing the Oxford Nanopore Technologies (ONT) libraries with ligation sequencing kit SQK-LSK109 using the ONT Genomic DNA by Ligation (SQK-LSK 109) protocol. Nanopore sequencing was performed for $48 \mathrm{~h}$ for each isolate on a separate MinION R9.4.1 flow-cell using ONT protocols. We converted the initially generated FAST5 raw sequence files to fastq sequence files with the Guppy Basecalling software ONT version 2.3.1 (Wick et al. 2019). We discarded sequences with a minimum quality read score of less than 7, and that were shorter than 1,000 bases to improve the assembly for further analysis. The quality filtered data shown in Table 1 for all four isolates were assembled using the high-noise single-molecule sequencing assembler Canu version 1.8 (Koren et al. 2017). We developed whole genome sequence (WGS) assemblies for Fusarium oxysporum f. sp. vasinfectum FOV1, FOV4, FOV5, and FOV8 using Nanopore sequencing (MinION) technology and then assembled the continuous contigs. This resulted in estimated genome sizes for FOV1 $52 \mathrm{Mb}$, FOV3 $68 \mathrm{Mb}$, FOV4 $68 \mathrm{Mb}$ and FOV8 $55 \mathrm{Mb}$ with over 90\% genome completeness. These genome assembly completeness statistics were generated with Benchmarking Universal Single-Copy Orthologs completeness analyses (single copy gene set called BUSCO) (Simao et al. 2015).

We polished the assembled contigs generated with MinION HTS with both ONT reads itself for any mis-assembly and error bases and with the quality sequences of Illumina reads using Burrows-Wheeler Aligner (BWA; ver. 0.7.17) with parameters "-x ont2d", and these were further polished using Pilon (Walker et al. 2014; Li and Durbin 2009). Finally we evaluated the assembled contigs with a quality assessment tool for genome assemblies known as BUSCO (Simao et al. 2015) and CEGMA with core eukaryotic genes (CEG) reference gene set (Parra et al. 2007).

We trained the gene prediction model for AUGUSTUS prediction for closest related species Fusarium oxysporum f. sp. lycopersici as a model organism to predict numbers of genes in our F. oxysporum f. sp. vasinfectum genomes (Ma et al. 2010). We found 16,263 genes in FOV1, 20,259 genes in FOV4, 20,375 genes in FOV5, and 16,615 genes in FOV8 (Hoff and Stanke 2013; Stanke and Waack 2003). The FOV1 and FOV4 results corresponds to the size assembled for particular races and these genome size and gene prediction were very much similar to recently reported genomes in this groups (Seo et al. 2019). We used a BLAST pipeline analyses (Srivastava et al. 2014) to identify unique genes for each of the races and found 525 
Table 1. Whole genome assembly statistics of four Fusarium oxysporum f. sp. vasinfectum races $1,4,5$, and 8 using MinlON reads and Canu assembler $^{\mathrm{a}}$

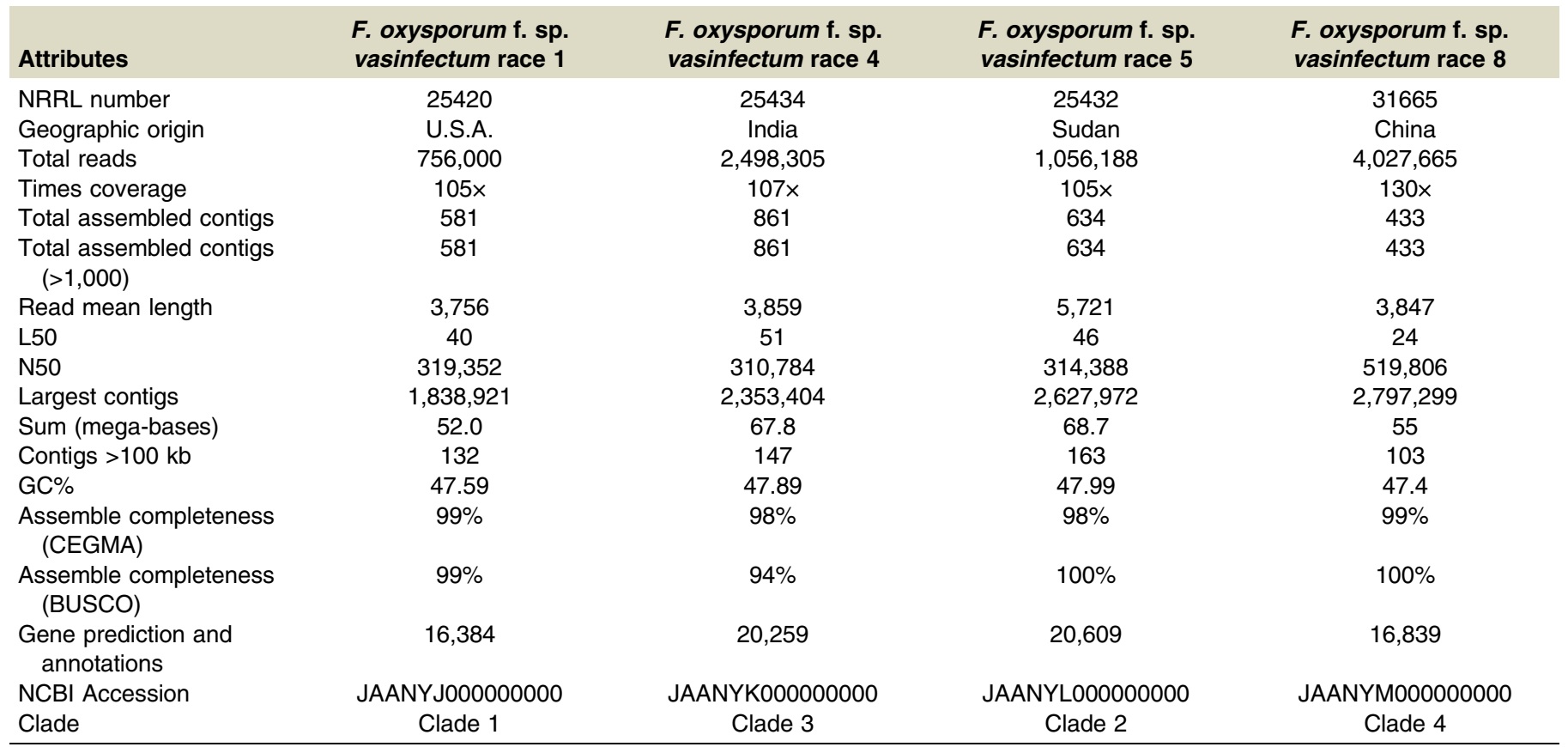

a CEGMA, core eukaryotic genes mapping approach (core eukaryotic gene set). BUSCO, benchmarking universal single-copy orthologs (single copy gene set).

unique genes in FOV1, 570 in FOV4, 1,242 in FOV5, and 383 in FOV8. We are currently evaluating these unique genes as candidates for diagnostic marker and methods development to permit rapid differentiation of $F$. oxysporum f. sp. vasinfectum subgroups. We assume that these genomes will provide a necessary resource for comparative genomic to identify genes or genome features that are involved in pathogenicity as well as different regulatory and diagnostics applications.

Accession numbers. These WGS Shotgun project has been deposited as GenBank project PRJNA605737, FOV1 accession number JAANYJ000000000, FOV4 accession number JAANYK000000000, and FOV5 accession number JAANYL000000000, and FOV8 JAANYM000000000 with SRA submission under project PRJNA605737.

\section{Acknowledgments}

We thank the ARS Culture Collection (NRRL) in Peoria for providing the reference isolates that we have used for this work.

\section{Literature Cited}

Armstrong, G. M., and Armstrong, J. K. 1960. American, Egyptian, and Indian cotton wilt Fusaria: Their pathogenicity and relationship to other wilt Fusaria. U.S. Dep. Agric. Tech. Bull. 1219.

Davis, R. M., Colyer, P. D., Rothrock, C. S., and Kochman, J. K. 2006. Fusarium wilt of cotton: Population diversity and implications for management. Plant Dis. 90:692-703.

Halpern, H. C., Bell, A. A., Wagner, T. A., Nichols, R. L., Olvey, J., Woodward, J. E., Sanogo, S., Jones, C. A., Chan, C. T., and Brewer, M. T. 2018. First report of Fusarium wilt of cotton caused by Fusarium oxysporum f. sp. vasinfectum race 4 in Texas, U.S.A. Plant Dis. 102:446.

Hoff, K. J., and Stanke, M. 2013. WebAUGUSTUS-a web service for training AUGUSTUS and predicting genes in eukaryotes. Nucleic Acids Res. 41: W123-W128
Kim, Y., Hutmacher, R. B., and Davis, R. M. 2005. Characterization of California isolates of Fusarium oxysporum f. sp. vasinfectum. Plant Dis. 89:366-372.

Koren, S., Walenz, B. P., Berlin, K., Miller, J. R., Bergman, N. H., and Phillippy, A. M. 2017. Canu: Scalable and accurate long-read assembly via adaptive k-mer weighting and repeat separation. Genome Res. 27:722-736.

Li, H., and Durbin, R. 2009. Fast and accurate short read alignment with BurrowsWheeler transform. Bioinformatics 25:1754-1760.

Ma, L. J., Van Der Does, H. C., Borkovich, K. A., et al. 2010. Comparative genomics reveals mobile pathogenicity chromosomes in Fusarium. Nature 464: 367-373.

Parra, G., Bradnam, K., and Korf, I. 2007. CEGMA: A pipeline to accurately annotate core genes in eukaryotic genomes. Bioinformatics 23 : 1061-1067. 
Rivera, Y., Zeller, K., Srivastava, S., Sutherland, J., Galvez, M., Nakhla, M., Poniatowska, A., Schnabel, G., Sundin, G., and Abad, Z. G. 2018. Draft genome resources for the phytopathogenic fungi Monilinia fructicola, M. fructigena, M. polystroma, and $M$. laxa, the causal agents of brown rot. Phytopathology 108:1141-1142.

Seo, S., Pokhrel, A., and Coleman, J. 2019. The genome sequence of five genotypes of Fusarium oxysporum f. sp. vasinfectum: A resource for studies on Fusarium wilt of cotton. Mol. Plant-Microbe Interact. 33:138-140.

Simao, F. A., Waterhouse, R. M., Ioannidis, P., Kriventseva, E. V., and Zdobnov, E. M. 2015. BUSCO: Assessing genome assembly and annotation completeness with single-copy orthologs. Bioinformatics 31:3210-3212.

Skovgaard, K., Nirenberg, H. I., O'Donnell, K., and Rosendahl, S. 2001. Evolution of Fusarium oxysporum $\mathrm{f} . \mathrm{sp}$. vasinfectum races inferred from multigene genealogies. Phytopathology 91:1231-1237.
Srivastava, S. K., Huang, X., Brar, H. K., Fakhoury, A. M., Bluhm, B. H., and Bhattacharyya, M. K. 2014. The genome sequence of the fungal pathogen Fusarium virguliforme that causes sudden death syndrome in soybean. PLoS One 9:e81832.

Stanke, M., and Waack, S. 2003. Gene prediction with a hidden Markov model and a new intron submodel. Bioinformatics 19(suppl. 2)::):i215-ii225.

Walker, B. J., Abeel, T., Shea, T., Priest, M., Abouelliel, A., Saktikumar, S., Cuomo, C. A., Zeng, Q., Wortman, J., Young, S. K., and Earl, A. M. 2014. Pilon: An integrated tool for comprehensive microbial variant detection and genome assembly improvement. PLoS One 9:e112963.

Wick, R. R., Judd, L. M., and Holt, K. E. 2019. Performance of neural network basecalling tools for Oxford Nanopore sequencing. Genome Biol. 20: 129. 